\title{
Hedging arguments
}

\author{
Erin Zaroukian \& Lyn Tieu* \\ LSA Annual Meeting, Portland, January 8-11, 2015
}

1. Introduction. Hedges such as loosely speaking and sorta indicate a mismatch between what is said and what is actually meant. As demonstrated by the example in (1), sorta is often used when a speaker doesn't know a more appropriate word or phrase at the time of utterance (Anderson 2014).

(1) I was running on concrete and accidentally sorta kicked the ground - that is to say, I didn't really kick the ground, but it was like kicking the ground. (Anderson 2014:02, ex.2)

In this study, we investigated the readings that arise from sorta-hedging. We present results indicating the possibility of hedging objects, verbs, and whole sentences, and we show that verb type, definiteness of the object, and stress on sorta all influence the availability of an object hedge reading.

2. Background. The hedge sorta modifies verb phrases and acts as an approximative. A natural paraphrase of sentences like (1) expresses that "sorta VERB" is similar to but not "VERB" in some respect (Anderson 2014). We will refer to this interpretation as the verb hedge reading, as it is the meaning of the verb that is approximated by sorta. According to Anderson (2014), certain verbs, namely intensional transitive verbs (ITV) and verbs of creation, additionally allow an 'object hedge' reading, where sorta hedges the direct object (see examples (2) and (3) from Anderson 2014). That the availability of the object hedge reading is restricted to certain classes of verbs is evidenced by the contrast between (2)-(3) and (4).

(2) I'm sorta looking for a horse.

Intensional transitive verb

a. I'm only half-heartedly looking for a horse.

(Verb hedge)

b. I'm looking for something like a horse.

(Object hedge)

(3) The carpenter sorta built a barn.

Creation verb

a. The carpenter did something that was like building a barn (e.g., putting together a prefabricated structure).

(Verb hedge)

b. The carpenter built something like a barn (e.g., a shed).

(Object hedge)

The soccer player sorta kicked a ball.

a. The soccer player did something that was like kicking to a ball.

(Verb hedge)

b. *The soccer player kicked something that was like a ball. (Object hedge unavailable)

Anderson also reports that only indefinite objects may be hedged. He proposes an analysis of hedging using Morzycki's (2011) alternative semantics implementation of Lasersohn's (1999)

\footnotetext{
*We are grateful to Alexandre Cremers, Lilia Rissman, Jérémy Zehr, and attendees of the 2015 LSA meeting for helpful feedback and discussion. The research leading to these results has received funding from the European Research Council under the European Union's Seventh Framework Programme (FP/2007-2013) / ERC Grant Agreement n.313610 and was supported by ANR-10-IDEX-0001-02 PSL* and ANR-10-LABX-0087 IEC. Authors: Erin Zaroukian (egz@jhu.edu) and Lyn Tieu (lyn.tieu@gmail.com), École Normale Supérieure, PSL Research University, Laboratoire de Sciences Cognitives et Psycholinguistique, CNRS, Paris.
} 
pragmatic halos. In brief, sorta approximates via slack regulation. It reduces the degree of precision with which an expression is interpreted by expanding the set of its possible referents (i.e. its 'resemblance alternatives'). A verb such as swim, for example, activates a set of resemblance alternatives such as float and wade. Expressions are interpreted relative to a degree of precision (through the PREC operator) - the larger the degree of precision, the smaller the set of resemblance alternatives; the smaller the degree of precision, the larger the set of alternatives. Sorta serves to lower this degree of precision. For object hedging, sorta modifies the object indirectly; through pointwise Hamblin functional application, the resemblance alternatives of the object project. To explain the restriction of object hedging to ITV/CREATION verbs, Anderson suggests that these verbs take property-type arguments, and that the lexical semantics of such verbs allows alternatives to project. To restrict hedging to indefinite objects, he treats the definite determiner the as a choice function that yields only a single alternative.

3. Current study. We designed an experiment to collect judgments on the available readings for sorta-hedging with three goals in mind. First, we aimed to collect systematic judgments that would provide more robust evidence of the availability of the multiple readings, as well as test for Anderson's definiteness and verb type effects. Second, we set out to test for a third possible reading that arises most naturally when sorta is deaccented (5); on this 'whole sentence hedge', the speaker appears to distance herself from the assertion.

Jane sorta broke the vase.

(The speaker doesn't want to fully admit what Jane did, perhaps because it will get Jane in trouble.)

(Whole sentence hedge)

Finally, we aimed to investigate the role of stress on sorta in modulating access to the object hedge reading.

4. Experiment. We used a $2 \times 2 \times 2$ within-subject design with three factors: Verb type (ITV/CREATION vs. OTHER), Definiteness (definite vs. indefinite), and Stress (stressed sorta vs. unstressed sorta). We tested 25 adult native speakers of English using a web-based multiple choice task. Before beginning the task, participants saw a set of instructions that included an explanation of hedging, as well as examples of object, verb, and whole sentence hedging. Instructions indicated that stressed "sorta" would appear in bold, large font (as in Fig. 1), while unstressed "sorta" would appear in smaller font (sorta). On each trial, participants had to choose the most likely hedge from among three options; these hedge options were presented in randomized order. Each participant saw a randomized order of 24 trials, which crossed all factors (verb type, definiteness, and stress).

5. Results. The results are presented in Figure 2. These results indicate the existence of the three kinds of hedge readings. To test whether verb type, definiteness, and stress had a statistically significant effect, we modeled the probability of selecting the object hedge option. The best fitting model included Participant as a random effect, and main effects of Verb (ITV/CREATION Vs. OTHER), Definiteness (definite vs. indefinite), and Stress (stressed vs. unstressed). Participants were more likely to choose the object hedge when the sentence contained an ITV or creation verb $(\beta=-.37$, $\mathrm{SE}=.03, \mathrm{p}<.001)$, when the object noun phrase was indefinite $(\beta=-.53, \mathrm{SE}=.03, \mathrm{p}<.05)$, and when sorta was stressed $(\beta=-.37, \mathrm{SE}=.03, \mathrm{p}<.001)$. Finally, while Anderson (2014) predicts a Verb $x$ 
Definiteness interaction (i.e. object hedging should only be possible with ITV/CREATION verbs taking indefinite objects), our results revealed no significant interactions.

\section{Charlie sorta fell in a pit. \\ What is the speaker hedging?}

1. "a pit": e.g., What Charlie fell in was actually more like a medium-size hole.

2. "fell in": e.g., Charlie actually just got his foot caught in it.

3. whole sentence: e.g., The speaker is trying not to fully admit what Charlie did, perhaps to avoid upsetting the person the speaker is talking to.

Figure 1: An example trial.

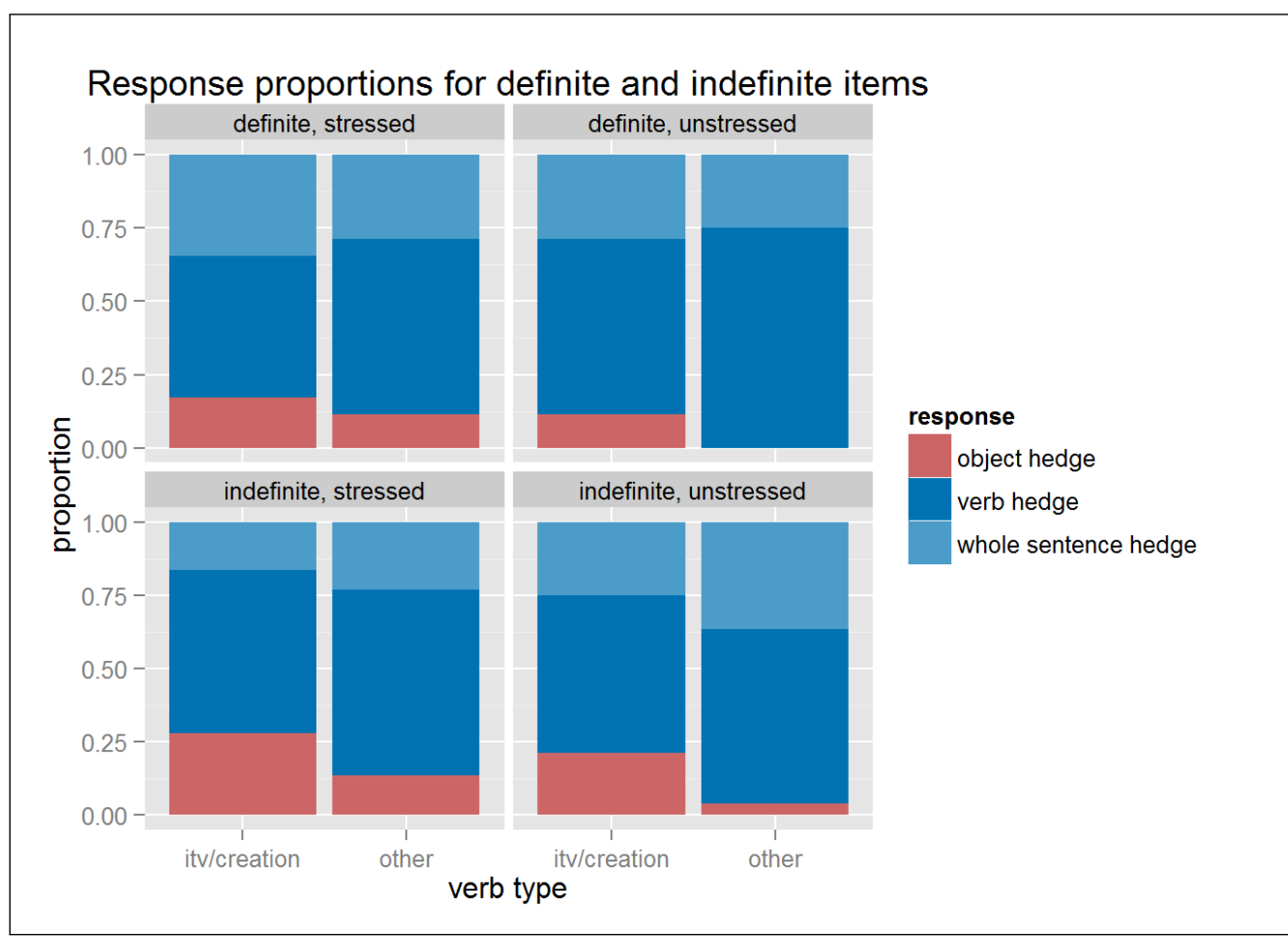

Figure 2: Proportions of object, verb, and whole sentence hedge responses across conditions.

6. Discussion. Our findings provide novel evidence for the availability of three hedge readings of sentences containing sorta. The results also suggest a role of verb type, definiteness, and stress, in modulating access to the object hedge reading. While it is easier to access the object hedge reading with ITV/CREATION verbs and indefinite objects, however, the results show that it is sometimes possible to access this reading with non-ITV/CREATION verbs and definite objects. This suggests that there is no strict grammatical constraint against hedging with non-ITV/CREATION verbs or definite objects. Instead, we speculate that what conditions the availability of object hedging is very simply the plausibility of the hedged object reading; the plausibility of the reading in turn 
hinges on the semantics of the verb. In particular, creation verbs make the object hedge reading very plausible, e.g., it is rather easy to imagine loosening the requirements on what counts as a barn when the average person is building a barn. As for the facilitatory effect of stress on access to the object hedge reading, we suggest that stress facilitates the activation of the object's resemblance alternatives, highlighting the approximative or 'loosened' sense of the object. This may be similar to the observed effect of focusing any, which is claimed to widen the domain of quantification such that individuals that would otherwise be considered exceptions count as part of the domain (Kadmon \& Landman 1993; Krifka 1995). In this case, stressed sorta triggers the expansion of the set of resemblance alternatives to include less prototypical instantiations of the object.

\section{References}

Anderson, Curt. 2014. Hedging verbs and nouns using an alternative semantics. In Martin Kohlberger, Kate Bellamy \& Eleanor Dutton (eds.), ConSOLE XXI: Proceedings of the 21st Conference of the Student Organization of Linguistics in Europe, 1-20. Leiden: Leiden University Centre for Linguistics.

Kadmon, Nirit \& Fred Landman. 1993. Any. Linguistics and Philosophy 16(4). 353-422.

Krifka, Manfred. 1995. The semantics and pragmatics of polarity items. Linguistic Analysis 25. 209-257.

Lasersohn, Peter. 1999. Pragmatic halos. Language 75(3). 522-551.

Morzycki, Marcin. 2011. Metalinguistic comparison in an alternative semantics for imprecision. Natural Language Semantics 19(1). 39-86. 